Y. Tsukamoto

Nagoya Math. J.

Vol. 52 (1973), 35-38

\title{
ÜBER GEWISSE RIEMANNSCHE MANNIGFALTIGKEITEN MIT POSITIVER KRÜMMUNG
}

\author{
Professor Minoru Kurita zum 60. Geburtstag gewidet \\ YÔTARO TSUKAMOTO*
}

Es ist wichtig und interessant, die Beziehungen zwischen Krümmung, Volumen, Geodätischen und topologischen Strukturen von Riemannschen Mannigfaltigkeiten mit positiver Krümmung zu untersuchen.

Die folgende Sätze sind wohlbekannt.

SATZ A (Heim [4]). Es sei $M$ eine vollständige Riemannsche Mannigfaltigkeit der Dimension $n \geqq 2$ mit Riemannscher Krümmung $K \geqq 1$ und $\operatorname{vol} M \geqq \frac{1}{2} \operatorname{vol} S^{n}$, wobei $S^{n}$ eine Sphäre mit konstanter Krümmung 1 ist und vol $M$ das Volumen von $M$ ist. Dann sind alle periodischen Geodätischen nicht kürzer als $\pi$.

SATZ B (Nagayoshi und Tsukamoto [5]). Es sei M eine vollständige Riemannsche Mannigfaltigkeit der Dimension $n \geqq 2$ mit Riemannscher Krümmung $K \geqq 1$ und $\operatorname{vol} M \geqq \frac{1}{2} \operatorname{vol} S^{n}$. Dann ist $M$ eine Homotopiesphäre oder isometrisch zum reellen projektiven Raum mit konstanter Krümmung 1.

Hier beweisen wir den folgenden Satz.

SATZ. Es sei $M$ eine vollständige Riemannsche Mannigfaltigkeit der Dimension $n \geqq 2$ mit Riemannscher Krümmung $K \geqq 1$ und vol $M \geqq \frac{1}{2} \operatorname{vol} S^{n}$. Ferner gebe es eine periodische Geodätische minimaler Bogenlänge $\pi$. Dann ist $M$ isometrisch zum reellen projektiven Raum mit konstanter Krümmung 1.

\section{Vorbereitungen}

(a) Volumen (vgl. Berger-Gauduchon-Mazet [2]). Es sei $M$ eine kompakte Riemannsche Mannigfaltigkeit, $g$ die Riemannsche Metrik von

Received October 3, 1972.

*Von Sonderforschungsbereich "Theoretische Mathematik der Universität Bonn" unterstüzt. 
$M$ und $v_{g}$ das Volumenelement von $M$. Für $m \in M$ hat man das Volumenelement $v_{m}$ vom Tangentialraum $M_{m}$ an $m$ in $M$. Es sei $\exp _{m}$ die Exponentialabbildung von $M_{m}$ in $M$ und $U_{m}$ die maximale offene Umgebung um 0 von $M_{m}$, die $\exp _{m}$ diffeomorph auf ihr Bild abbildet. Die Umgebung $U_{m}$ heisst die injektive Umgebung von $m$. Für jeden Punkt $x \in U_{m}$ sei $\theta(x)$ die Jacobische Determinante von $\exp _{x}$. Dann haben wir

$$
\operatorname{vol} M=\int_{U_{m}} \theta \cdot v_{m}
$$

Wir verwenden Jacobifelder, um Volumen zu berechnen. Es sei $U_{\varepsilon}(m)$ eine normale Umgebung vom Radius $\varepsilon>0$ um $m$ in $M$. Für $0<r<\varepsilon$ und Einheitsvektor $u$ auf $U_{\varepsilon}(m)$ geben wir den Ausdruck von $\theta(r u)$. Es sei $c_{u}$ die Geodätische von $m$ mit der Anfangsrichtung $u$. Es sei $\left\{y_{2}, \cdots, y_{n}\right\}$ eine orthonormale Basis vom orthogonalen Komplement $u^{\perp}$ von $u$. Es sei $Y_{i}(s), i=2, \cdots, n$, Jacobifelder längs der Geodätischen $c_{u}$, so dass $Y_{i}(0)=0$ und $Y_{i}^{\prime}(0)=y_{i} / r$, wobei $Y^{\prime}$ die kovariante Ableitung von $Y_{i}$ in der Richtung $\dot{c}_{u}$ ist. Dann haben wir

$$
\theta(r u)=\operatorname{det}\left(\left\langle Y_{i}(r), Y_{j}(r)\right\rangle\right)^{1 / 2}, \quad i, j=2, \cdots, n,
$$

wobei $\langle$,$\rangle das innere Produkt von M$ ist.

(b) Die Vergleichssätze von Rauch und Toponogov (vgl. GromollKlingenberg-Meyer [3]).

Für $m \in M$ bezeichne man mit $G_{m}$ die Menge aller 2-dimensionalen linearen Teilräume von $M_{m}$ und $G_{M}:=\bigcup_{m \in M} G ; c:[0, l] \rightarrow M$ eine differenzierbare Kurve. Für $t \in[0, l]$ bezeichne man mit $G_{c, t}$ die Menge aller 2-dimensionalen linearen Teilräume $\sigma \subset M_{c(t)}$ mit dem Tangentenvektor $\dot{c}(t) \in \sigma$ und $G_{c}:=\bigcup_{t \in[0, l]} G_{c, t}$. Man kann jeder Ebene $\sigma \in G_{M}$ die Riemannsche Krümmung $K_{\sigma}=K(u, v)$ zuordnen, indem man linear unabhängige Vektoren $u, v \in \sigma$ wählt.

VERGLEICHSSATZ VON RAUCH. Es sei $S^{n}$ eine n-dimensionale Sphäre mit konstanter Krümmung 1, $M$ eine $n$-dimensionale Riemannsche Mannigfaltigkeit $(n \geqq 2) ; c:[0, l] \rightarrow M, \tilde{c}:[0, l] \rightarrow S^{n}$ normale Geodätische, $Y, \tilde{Y}$ Jacobifelder längs $c$, $\tilde{c}$ mit $Y(0)=0, \tilde{Y}(0)=0$ und $\left\langle Y^{\prime}, \dot{c}\right\rangle(0)=\left\langle\tilde{Y}^{\prime}, \dot{\tilde{c}}\right\rangle(0)$ $=0$ sowie $\left\|Y^{\prime}(0)\right\|=\left\|\tilde{Y}^{\prime}(0)\right\|$. c habe keine konjugierten Punkte in $(0, l)$. Ist dann die Krümmung von $M$ längs $c$ nicht kleiner als 1 , d. h. $K_{\sigma} \geqq 1$ für alle $\sigma \in G_{c(t)}$ und alle $t \in[0,1]$, so gilt $\|Y(t)\| \leqq\|\tilde{Y}(t)\|, t \in[0, l]$. 
Haben wir $\left\|Y\left(t_{0}\right)\right\|=\left\|\tilde{Y}\left(t_{0}\right)\right\| \neq 0$ für $t_{0} \in[0, l]$, so gilt $K(Y(t), \dot{c}(t))=1$ für alle $t \in\left[0, t_{0}\right]$.

VERGLEICHSSATZ VON TOPONOGOV. Es sei $M$ eine vollständige Riemannsche Mannigfaltigkeit der Dimension $n$ ( $n \geqq 2$ ), und $K_{\sigma} \geqq k>0$ für alle $\sigma \in G_{M}$. Es sei pqr ein geodätisches Dreieck auf $M$ und $\tilde{p} \tilde{q} \tilde{r}$ ein geodätisches Dreieck auf $S^{n}(k)$ mit $d(\tilde{p}, \tilde{q})=d(p, q), d(\tilde{p}, \tilde{r})=d(p, r)$ und dem Winkel $\Varangle \tilde{p}=\Varangle p$. Dann ist $d(q, r) \leqq d(\tilde{q}, \tilde{r})$, (wobei $S^{n}(k)$ eine $n$-dimensionale Sphäre mit konstanter Krümmung $k$ ist und $d$ die Abstandsfunktion ist).

\section{Beweis von Satz}

Es sei $c$ eine periodische Geodätische minimaler Bogenlänge $\pi$. Es sei $m$ ein Punkt von $c$. Es sei $n$ ein beliebiger Punkt von $M$ und $r$ ein nächster Punkt auf $c$ von $n$. Dann haben wir $d(n, r) \leqq \pi / 2$ (vgl. Berger [1]), und eine minimale Geodätische von $n$ nach $r$ ist orthogonal zu $c$ in $r$. Wenn wir den Vergleichssatz von Toponogov verwenden und mit dem gleichen Argument wie im Beweis des Satzes von Berger ([5]) diskutieren, haben wir $d(m, n) \leqq \pi / 2$. Es sei $U_{m}$ die injektive Umgebung von $m$. Dann liegt $U_{m}$ im offenen Ball um 0 in $M$ mit Radius $\pi / 2$. Nach (1), (2), dem Vergleichssatz von Rauch und einer Eigenschaft von Grammscher Determinante haben wir

$$
\operatorname{vol} M \leqq \frac{1}{2} \operatorname{vol} S^{n} .
$$

Daher haben wir vol $M=\frac{1}{2} \operatorname{vol} S^{n}$. Nach (1), (2) und dem Vergleichssatz von Rauch stimmt $U_{m}$ mit dem offenen Ball vom Radius $\pi / 2$ um 0 in $M_{m}$ überein und die Menge $\exp _{m}\left(U_{m}\right)$ ist isometrisch zur Hemisphäre von $S^{n}$. So ist $M$ ein Raum mit konstanter Krümmung 1 . Deshalb ist $M$ isometrisch zu einem reellen projektiven Raum mit konstanter Krümmung 1 , weil vol $M=\frac{1}{2} \operatorname{vol} S^{n}$ ist.

\section{LITERATUR}

[1] M. Berger: Les variété riemannienne à courbure positive, Bull,, Soc., Belgique. 10, 89-104 (1958).

[2] M. Berger, P. Gauduchon und E. Mazet: Le spectre d'une variété riemannienne, Springer-Verlag. Lecture notes 194, 1971.

[ 3 ] D. Gromoll, W. Klingenberg und W. Meyer: Riemannsche Geometrie im Grossen, Springer-Verlag. Lecture notes 55, 1968.

[4] C. Heim: Une borne pour la longueur des géodésiques périodiques d'une variété riemannienne compacte, These. Université Paris. 1971. 
[5] T. Nagayoshi and Y. Tsukamoto: On positively curved Riemannian manifolds with bounded volume, Tohoku Math. J., 25, 213-218 (1973).

Mathematisches Institut

Universität Kyushu 